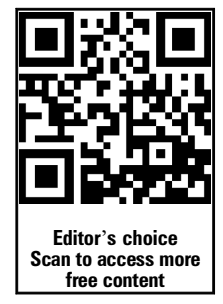

Upper GI/Bariatric Surgery, University Hospital of North Tees, Stockton-on-Tees, UK

\section{Correspondence to}

M J Courtney, Upper GI/Bariatric Surgery, University Hospital of North Tees, Hardwick Road, Stockton-on-Tees, UK; mjcourtney01@doctors.org.uk

Received 24 February 2014 Revised 16 March 2014 Accepted 17 March 2014 Published Online First 3 April 2014
CrossMark

To cite: Courtney MJ, Rao M, Teasdale R, et al. Frontline Gastroenterology 2014:5:272-276.

\title{
Would you have laparoscopic Nissen fundoplication again? A patient satisfaction survey in a UK population
}

\section{Michael J Courtney, Milind Rao, Rebecca Teasdale, Rajesh Jain, Bussa Gopinath}

\begin{abstract}
Objective Laparoscopic Nissen fundoplication (LNF) effectively reduces objective gastrooesophageal reflux. It can however cause side effects which affect quality of life or fail to improve subjective reflux symptoms. This study aims to assess patient satisfaction following LNF by assessing whether patients would have the procedure again.
\end{abstract}

Design Telephone survey using a structured questionnaire. Participation was voluntary.

Setting UK Foundation Trust (two university hospitals).

Patients All patients who had LNF performed by a single surgeon between November 2008 and June 2012

Main outcome measures Primarily, current reflux symptoms, antiacid medication requirement and whether participants would choose to have the procedure again (should they still have their initial symptoms). Further measures were conversion to open procedure, need for redo or reversal, and mortality.

Results 99 patients underwent LNF in the quoted period; 71 were contactable and willing to participate. Of the 99 , two required redo operations (neither of whom was contactable), and one had a reversal (primary operation included). Median time since the operation was 33 months (range 5-48 months). Compared with preoperatively, $72 \%$ rated their current refluxsymptom severity as $\leq 2 / 10,23 \%$ as $3-6 / 10$ and $4 \%$ as $7-10 / 10$. $75 \%$ were not taking any antiacid medication. $89 \%$ of patients said that they would have the procedure again.

Conclusions This study provides supporting evidence that LNF improves reflux symptoms and decreases medication use at intermediate-term follow-up. These results will aid counselling and reassurance of patients regarding the risks and benefits of LNF as the majority of postoperative patients were sufficiently satisfied to choose the operation again.

\section{BACKGROUND}

Gastro-oesophageal reflux disease (GORD) is a common condition with an estimated prevalence in the Western world of $10 \%$ $20 \%$ and incidence of five per 1000 person-years. ${ }^{1}$ The management of GORD is multi-disciplinary, often involving general practitioners, gastroenterologists, surgeons and specialist nurses, all of whom should have an awareness of the pros and cons of each management option. The recently published Royal College of Surgeons of England (RCSE) Commissioning Guide: Gastro-oesophageal Reflux Disease ${ }^{2}$ outlines the indications for surgery in patients with GORD. Surgery should be considered in those with proven objective reflux (through 24-h $\mathrm{pH}$ monitoring), in whom other pathologies have been excluded (including malignancy, eosinophilic oesophagitis and motility disorders) and who have one of the following indications:

1. Volume reflux, especially affecting sleep, or during physical activities that involve stooping

2. Breakthrough symptoms of heartburn despite optimal medical therapy

3. Intolerance of proton pump inhibitors

4. Patient preference to avoid lifelong medication

5. Postprandial chest pain or dysphagia from incarcerated para-oesophageal hernia

6. Atypical symptoms such as aspiration, cough or hoarse voice if confirmed on $\mathrm{pH}$ testing. 
Although multiple variants of antireflux operations are described, laparoscopic Nissen fundoplication (LNF) is currently the procedure of choice for the surgical management of GORD. ${ }^{3}$ This is reiterated in the RCSE guidance, which recommends fundoplication for the surgical management of GORD. When considering the option of surgical management of GORD, and particularly during preoperative discussion and for informed consent, it is necessary to explain to the patient (along with potential benefits) the potential adverse outcomes of surgery including side effects, complications and failure to achieve a desired aim. ${ }^{4}$ With LNF, the desired aim is ultimately an improvement in quality of life (in this case through decreased reflux symptoms and medication use). Objective outcomes such as oesophageal $\mathrm{pH}$, manometry and medication requirement are repeatable measures that can assess the technical success of the procedure but do not necessarily assess (or represent) the extent of symptoms that patients are experiencing since the surgery or the impact that it has on their lives. ${ }^{5} 6$ Similarly, one must not underestimate the importance of different patients' experiences during their journey through surgery, and how it may influence their perception of the whole process. Exploring these subjective aspects of surgery is challenging, but ultimately the aim of surgery is to make the patient feel better in his/her entirety, and not only improvement in the presenting symptom. It is for these reasons we feel it important to explain the chances of 'satisfaction' following surgery in support of likelihood of specific outcomes.

In this study, the aim was to assess the satisfaction of LNF outcome in a UK population; specifically, whether they would have the procedure again.

\section{METHODS}

A retrospective search was performed of electronic theatre records to identify all patients for whom the intended procedure was primary LNF, performed by single surgeon (BG) in a UK NHS foundation trust. A telephone survey was then conducted of all consecutive patients by two hospital employees (both surgical trainees, but neither of whom was involved in the patients' management) over a 4-month period. Patients were informed that the survey was entirely voluntary. Using a structured questionnaire, the following questions were asked:

1. How are your reflux symptoms now compared with preoperatively $(0=$ no symptoms, $10=$ no better than preoperatively)?

2. Do you still use any antiacid medication? (and so including anything that the patient interpreted as remedy for symptoms, either prescribed or over the counter)

3. With the benefit of hindsight, would you have the procedure again?

No further questions were asked and answers were not elaborated on. In the event of a participant not being contactable or available, a second attempt was made at a later date. Further outcome measures (collected from electronic hospital records) were conversion to open procedure, need for redo or reversal, and mortality. Any patients refusing to participate were excluded.

\section{RESULTS}

A total of 99 patients underwent LNF over a 42-month period from November 2008 to May 2012, all of whom had either failure of medical management or opted for surgery in spite of it. All procedures were completed laparoscopically. A standard surgical technique was used similar to that described in published papers, ${ }^{7}$ and all included the Rossetti modification (ie, wrapping only the anterior fundus and not dividing the short gastric vessels). Median follow-up was 33 months (range 5-48). Overall, 71 (72\%) patients partook in the survey; the other $28(28 \%)$ were not contactable. No patient who was contacted refused to participate. At the time of follow-up, 3 (3\%) patients had required reoperation: one needed reversal (in the contactable group and so included in analysis) and two were redo procedures (both uncontactable). One patient had died during follow-up of a cause unrelated to surgery (figure 1).

Compared with preoperatively (if $0 / 10$ indicated no symptoms and 10/10 was as severe as preoperatively), $72 \%$ (51) rated their current reflux-symptom severity as $\leq 2 / 10,23 \%(16)$ as $3-6 / 10$ and $4 \%$ (3) as $7-10 / 10$. One patient was unsure of the symptom severity (figure 2). At the time of contacting, 21\% (15) were taking regular antiacid medication, $4 \%$ (3) on an as-required (PRN) basis, and 75\% (53) were not needing any antiacid medication at all (figure 3 ). Overall, $89 \%$ (63) of patients said that they would opt to have the procedure again if required (figure 4).

\section{DISCUSSION}

This study confirms in the studied population that LNF successfully reduces both reflux symptoms and

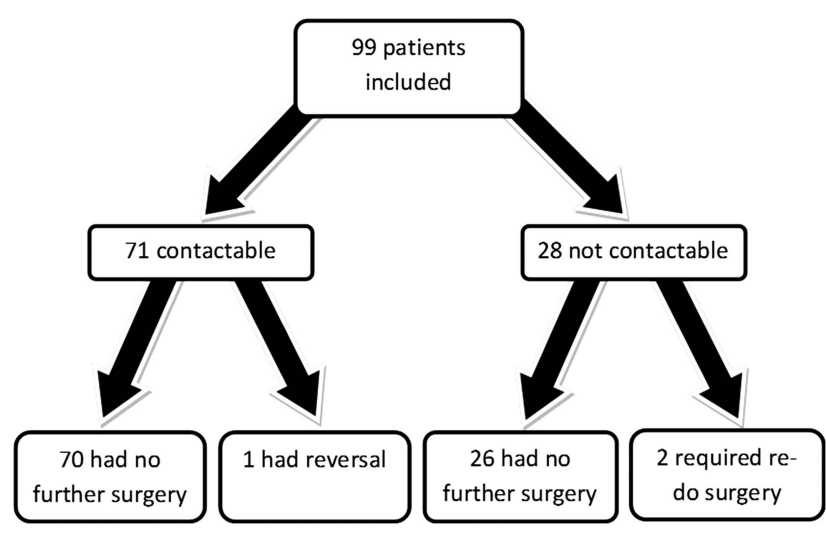

Figure 1 Flowchart outlining postoperative outcomes of patients in each group. 


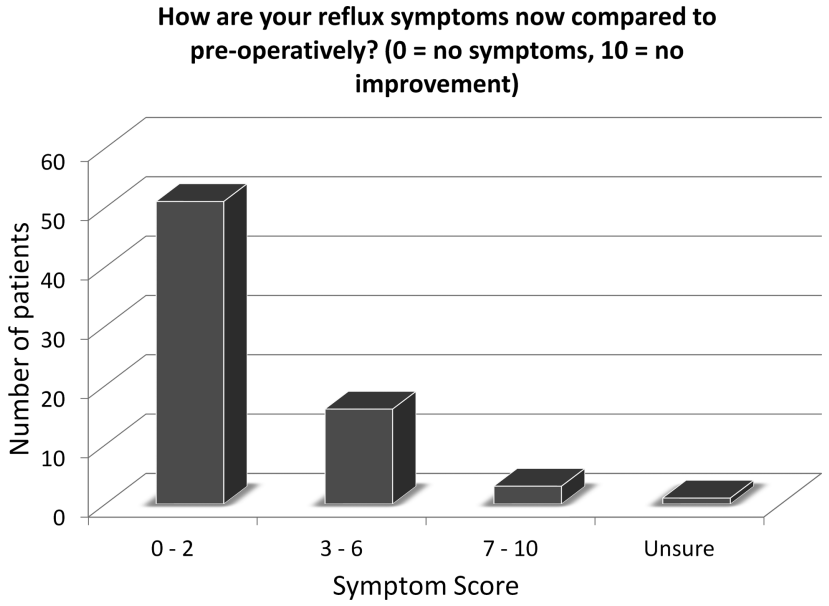

Figure 2 Breakdown of symptom scores.

antiacid medication requirement in the vast majority of patients at intermediate-term follow-up, and that $89 \%$ of participants would choose the operation again. This is important as currently there is a paucity of data in this area in the population, and so will provide information regarding subjective results of surgery and subsequently aid both clinicians and patients in the decision-making process.

Literature review using PubMed revealed 23 previous published studies that assessed whether patients would undergo LNF again (either themselves, or would recommend it to a friend or relative); this included all variants of laparoscopic fundoplication, all indications and studies whereby fundoplication was one arm only. There was a noticeable lack of data on the demographic group in our study, with only 12 previous studies from European countries, ${ }^{8-19}$ one of which was from the UK. ${ }^{17}$ Overall, the number that would recommend the procedure/have it again was $80 \%-98 \%$. The response rate of $72 \%$ achieved in our study is similar to all previous studies mentioned, and also in direct comparison with published large randomised control trials with prospective follow-up

\section{Do you still use any antiacid medication?}

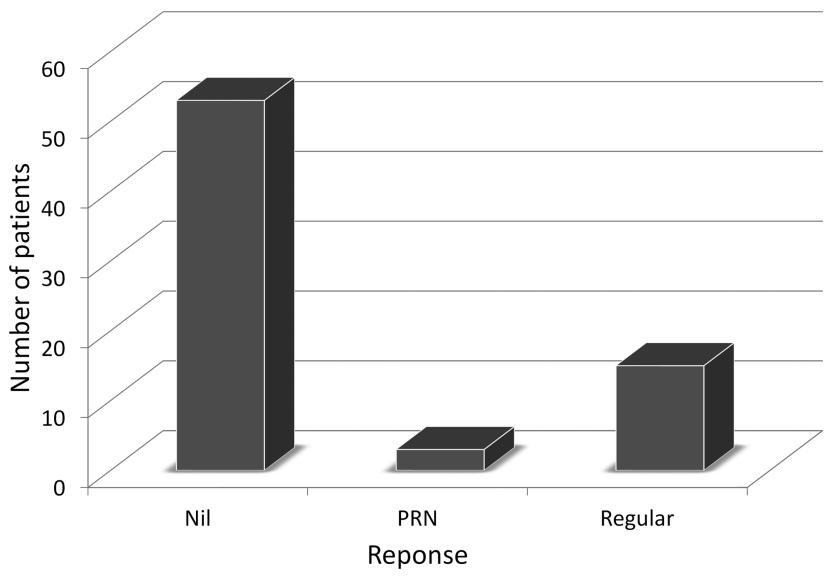

Figure 3 Breakdown of medication usage.

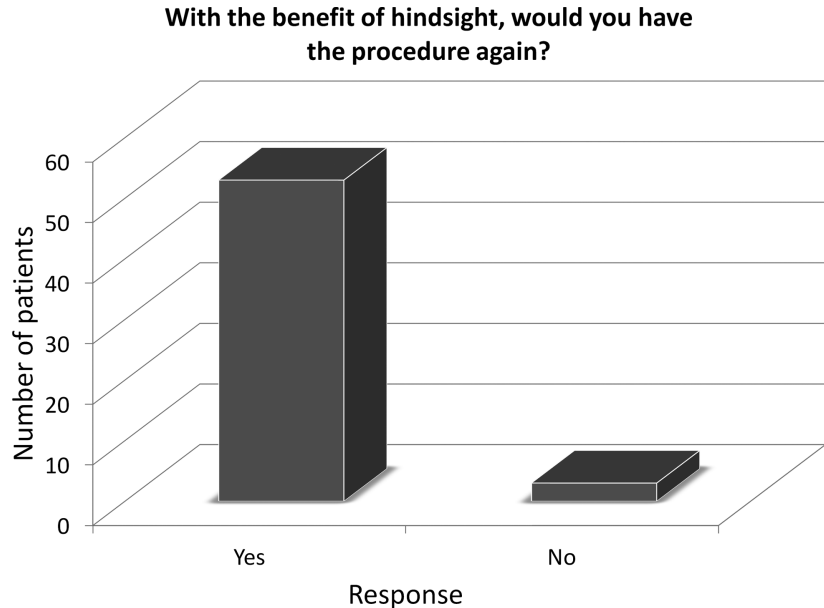

Figure 4 Breakdown of satisfaction.

(LOTUS trial, 73\% 5-year follow-up ${ }^{20}$ ). The number of participants and length of follow-up in our study are also in the range similar to others. With regard to follow-up, it is interesting to note that the duration since surgery in previous studies does not seem to reflect satisfaction. In direct comparison with the other UK study (comparing Toupet and Nissen fundoplication), the participant number was identical (99). Their design was different to ours being a paper-based questionnaire and participants being allocated to Toupet or Nissen according to the surgeon's preference (as opposed to all of the participants in our study having only Nissen). The response rate achieved in their study, however (63\%), was similar to ours (72\%). With regard to results, both their study and ours revealed decreases in medication use $(44 \%$ and $25 \%$, respectively), and overall high satisfaction, with $89 \%$ of our participants saying that they would chose the procedure again compared with $86 \%$ of their study. Comparison of our results with the much larger LOTUS trial again revealed similar outcomes; in this study 16\% requiring medication at 5 -year follow-up, with a symptom remission rate of $90 \%$ at 3 years, and $85 \%$ at 5 years. Our outcome of $3 \%$ reoperation rate was low in comparison with results published in a meta-analysis of high quality studies (6\%). ${ }^{21}$ It is however unclear whether this is due to patient selection, surgical expertise/experience or length of follow-up.

Although the response rate obtained was comparable with those previously published, it must be recognised as a limitation to this study. As the reason for lack of contact was always practical however (due to incorrect/out-of-date contact details, or 'no answer') we would not anticipate the results to differ between that group from the contactable one. We do, however, acknowledge that there were two patients who required redo surgery in the uncontactable group who presumably would have reported a poor result to the primary surgery. The use of telephone survey was chosen to increase response rate and allow for 
multiple attempts at obtaining responses. The chances of false answers being given were decreased by voluntary participation being explicitly stated and interviewers not being involved with patient care. Similarly, the only follow-up for patients following LNF is a telephone call 1 month postoperatively by a specialist nurse, and so all participants were beyond this and hence out of follow-up, and should not have worried that their care be affected by any answers given. In this study, we did not explore the reasons for negative responses (ie, would not have surgery again) as participants were no longer under follow-up, and we did not wish to ask them about symptoms or problems that may require investigation or follow-up and yet not be in a position to offer help. We also were not able to correlate postoperative findings with preoperative symptoms and medication use, and would consequently recommend further studies to use a validated health-related quality of life tool prospectively to assess, and hence compare, symptoms and outcomes preoperatively and postoperatively (as is now advised by the RCSE ${ }^{2}$ ). Previous studies have suggested side effects and persistent symptoms as major causes of dissatisfaction following LNF, ${ }^{22} 23$ and so further study on those in the group who would not undergo surgery again may be useful, especially to find any way to predict who is at risk of a worse outcome. We do not, however, feel that this additional information changes the primary outcome of this study.

The ultimate purpose of LNF for uncomplicated GORD is improvement in quality of life, hence the subjective outcomes that the patients experience (both positive and negative) are of paramount importance. This study is important due to the information it provides on these subjective outcomes. The benefits of LNF for uncomplicated GORD are decreased objective reflux, ${ }^{24}$ and hence decreased symptoms and medication (as evidenced by this study) and overall the potential for improvement in quality of life. Negative outcomes of LNF include new symptoms such as dysphagia, bloating and flatulence, along with ongoing/recurrence of symptoms. Prior to consideration of surgery (or referral for it), and as part of the counselling/consent process for all operations, there should be discussion regarding all of these outcomes, ideally with the ability to give likelihoods of each occurring. Postoperative negative outcomes are difficult to quantify and predict as 'failure' depends on definition used (eg, continued symptoms compared with need for reoperation) and whether using subjective or objective measures (eg, GORD symptoms vs endoscopically proven oesophagitis). Furthermore, previous studies have shown that postoperative reflux symptoms do not necessary correlate with objective reflux, and so do not always reflect technical success of the operation. ${ }^{5}{ }^{6}$ These factors combined make the preoperative discussion difficult for both clinician and patient, as it means weighing-up the odds of improvement in a symptom that a patient currently finds distressing with an (often subjective) outcome that the patient has not experienced before (eg, dysphagia or bloating), which also does not reflect on the technical success of the operation. Furthermore, different outcomes of surgery (eg, change of diet, need for medication) may have a greater impact on some patients than others and so perception of a 'positive' outcome may vary between different patients. We also must not forget the impact of the surgical journey on patients' lives, including preoperative and postoperative lifestyle changes, time off work and recovery. Consequently, although information about likelihood of individual risks is essential, it may be useful to explain to patients the chance of an overall 'satisfactory' outcome, which takes into account all aspects of surgery. We feel that the best indicator of this is whether or not patients (who have had surgery) would choose to have the procedure again.

The information that this study provides is therefore important for three reasons. First, it confirmed that the previously published benefits in reflux symptoms and medication use are valid in the UK population. Second, when counselling patients preoperatively, it provides a reference value for the likelihood of successful outcome in that $89 \%$ of people in a similar demographic group had such a successful outcome that they would have the procedure again. Finally, these data will allow a reference figure to monitor surgical outcomes as necessary for quality improvement. It is advisable that future patient reported outcomes should be monitored using the health-related quality of life instruments.

\section{CONCLUSIONS}

In all, 94\% of patients had improvement in their reflux symptoms post-LNF, 79\% were not requiring regular antiacid medication and overall $89 \%$ would have the procedure again. The results of this study will aid counselling and reassurance for patients regarding the risks and benefits of LNF.

\section{What is already known on this topic}

- Gastro-oesophageal reflux disease (GORD) is a common condition throughout the Western world.

- One of the (multidisciplinary) management options for GORD is laparoscopic Nissen fundoplication (LNF).

- Part of the preoperative discussion and decisionmaking process should focus on potential benefits and adverse outcomes of surgery. Subjective outcomes do not always correlate well with objective outcomes or technical success of surgery.

- International studies have reported high satisfaction following LNF but there is a lack of data available regarding the UK population. 


\section{What this study adds}

LNF successfully reduces reflux symptoms and antiacid medication use in the vast majority of the studied UK population at intermediate-length follow-up.

- In the studied population, $89 \%$ of patients were sufficiently satisfied with the outcome of the surgery that they would choose to have it again.

- The data will provide a useful reference for monitoring surgical outcomes in the future.

How might it impact on clinical practice in the foreseeable future

- This study will aid counselling and reassurance for patients regarding the risks and benefits of LNF as a management option for GORD.

- This study has highlighted the importance of prospective use of validated health-related quality of life tools to assess and compare preoperative and postoperative outcomes and to facilitate further study into reasons for dissatisfaction.

Contributors $\mathrm{MJC}$ was the main author of the manuscript, assisted throughout by MR. RT (along with MJC) contributed significantly to the data collection. RJ reviewed and assisted in final write-up of the manuscript. BG supervised the entire project.

Competing interests None.

Provenance and peer review Not commissioned; externally peer reviewed.

\section{REFERENCES}

1 Dent J, El-Seraq HB, Wallander MA, et al. Epidemiology of gastro-oesophageal reflux disease: a systematic review. Gut 2005;54:710-17.

2 Attwood S, Rasheed A, McKay C, et al. Royal College of Surgeons of England Commissioning Guide: Gastro-oesophageal reflux disease (GORD). 2013. http://www.rcseng.ac.uk/ healthcare-bodies/docs/published-guides/gord (accessed 12 Mar 2014).

3 Salminen P. The laparoscopic Nissen fundoplication-a better operation? Surgeon 2009;7:224-7.

4 General Medical Council. Consent: patients and doctors making decisions together. 2009. http://www.gmc-uk.org/ guidance/ethical_guidance/consent_guidance_index.asp (accessed 1 Sep 2013).

5 Yang H, Watson DI, Kelly J, et al. Esophageal manometry and clinical outcome after laparoscopic Nissen fundoplication. J Gastrointest Surg 2007;11:1126-33.

6 Galvani C, Fisichella PM, Gorodner MV, et al. Symptoms are a poor indicator of reflux status after fundoplication for gastroesophageal reflux disease: role of esophageal function tests. Arch Surg 2003;138:514-18.
7 Attwood SEA, Lundell L, Ell C, et al. Standardization of surgical technique in antireflux surgery: the LOTUS trial experience. World J Surg 2008;32:995-8.

8 Granderath FA, Kamolz T, Schweiger UM, et al. Quality of life and subjective evaluation of outcome quality 3 years after laparoscopic antireflux surgery. Chirurg 2000;71:950-4.

9 Heikkinen TJ, Haukipuro K, Bringman S, et al. Comparison of laparoscopic and open Nissen fundoplication 2 years after operation. A prospective randomized trial. Surg Endosc 2000;14:1019-23.

10 Kamolz T, Granderath FA, Schweiger UM, et al. Laparoscopic Nissen fundoplication in patients with nonerosive reflux disease. Long-term quality-of-life assessment and surgical outcome. Surg Endosc 2005;19:494-500.

11 Rosenthal R, Peterli R, Guenin MO, et al. Laparoscopic antireflux surgery: long-term outcomes and quality of life. J Laparoendosc Adv Surg Tech 2006;16:557-61.

12 Salminen PT, Laine SO, Ovaska JT. Late subjective results and symptomatic outcome after laparoscopic fundoplication. Surg Laparsc Endosc Percutan Tech 2006;16:203-7.

13 Vidal O, Lacy AM, Pera M, et al. Long-term control of gastroesophageal reflux disease symptoms after laparoscopic Nissen-Rosetti fundoplication. J Gastrointest Surg 2006;10:863-9.

14 del Genio G, Rossetti G, Brusciano L, et al. The NissenRossetti fundoplication: outcomes and lessons learned in 35 years experience with the same procedure. Minerva Chir 2007;62:1-9.

15 Salminen PT, Hiekkanen HI, Rantala AP, et al. Comparison of long-term outcome of laparoscopic and conventional nissen fundoplication: a prospective randomized study with an 11-year follow-up. Ann Surg 2007;246:201-6.

16 Fein M, Bueter M, Thalheimer A, et al. Less reflux recurrence following Nissen fundoplication: results of laparoscopic antireflux surgery after 10 years. Chirurg 2008;79:759-64.

17 Sgromo B, Irvine LA, Cuschierei A, et al. Long-term comparative outcome between laparoscopic total Nissen and Toupet fundoplication: Symptomatic relief, patient satisfaction and quality of life. Surg Endosc 2008;22:1048-83.

18 Kornmo TS, Ruud TE. Long-term results of laparoscopic Nissen fundoplication due to gastroesophageal reflux disease. A ten year follow-up in a low volume center. Scand J Surg 2008;97:227-30.

19 Salminen P, Karvonen J, Ovaska J. Long-term outcomes after laparoscopic Nissen fundoplication for reflux laryngitis. Dig Surg 2010;27:509-14.

20 Galmiche JP, Hatlebakk J, Attwood S. Laparoscopic antireflux surgery vs esomeprazole treatment for chronic GERD. The LOTUS Randomized Clinical Trial. JAMA 2011;305:1969-77.

21 Peters MJ, Mukhtar A, Yunas RM, et al. Meta-analysis of randomized clinical trials comparing open and laparoscopic anti-reflux surgery. Am J Gastroenterol 2009;104:1548-61.

22 Beenen E, Fogarty P, Roberts RH. Predicting patient dissatisfaction following laparoscopic Nissen fundoplication: an analysis of symptoms. Surg Endosc 2013;27:1579-86.

23 Humphries LA, Hernandez JM, Clark W, et al. Causes of dissatisfaction after laparoscopic fundoplication: the impact of new symptoms, recurrent symptoms, and the patient experience. Surg Endosc 2013;27:1537-45.

24 Stefanidis D, Hope WW, Kohn GP, et al. Guidelines for surgical treatment of gastroesophageal reflux disease. Surg Endosc 2010;24:2647-69. 